\title{
Designing 2D Phononic Crystal Slabs with Transmission Gaps for Solid Angle as well as Frequency Variation
}

\author{
Sven M. Ivansson \\ Department of Underwater Research, Swedish Defence Research Agency, FOI Kista, 16490 Stockholm, Sweden \\ Correspondence should be addressed to Sven M. Ivansson, sven.ivansson@foi.se \\ Received 29 January 2009; Accepted 15 June 2009 \\ Recommended by Mohammad Tawfik
}

\begin{abstract}
Phononic crystals (PCs) can be used as acoustic frequency selective insulators and filters. In a two-dimensional (2D) PC, cylindrical scatterers with a common axis direction are located periodically in a host medium. In the present paper, the layer multiplescattering (LMS) computational method for wave propagation through 2D PC slabs is formulated and implemented for general 3D incident-wave directions and polarizations. Extensions are made to slabs with cylindrical scatterers of different types within each layer. As an application, the problem is considered to design such a slab with small sound transmittance within a given frequency band and solid angle region for the direction of the incident plane wave. The design problem, with variable parameters characterizing the scatterer geometry and material, is solved by differential evolution, a global optimization algorithm for efficiently navigating parameter landscapes. The efficacy of the procedure is illustrated by comparison to a direct Monte Carlo method.
\end{abstract}

Copyright ( $) 2009$ Sven M. Ivansson. This is an open access article distributed under the Creative Commons Attribution License, which permits unrestricted use, distribution, and reproduction in any medium, provided the original work is properly cited.

\section{Introduction}

Just as photonic crystals can be used to manipulate light, phononic crystals (PCs) with inclusions in a lattice with single, double, or triple periodicity can be used to manipulate sound [1]. When a sound wave of a certain frequency penetrates the PC, the energy is scattered by the inclusions. According to Bragg's law, constructive and destructive interference appears in certain directions. It follows that transmission and/or reflection for certain frequencies can be absent (band gaps), even for all angles of incidence (absolute band gaps). The band gaps are ideal and fully developed only in space-filling PCs. In a PC slab with finite thickness, the "band-gap wavefields" are reduced significantly but they do not vanish throughout the gap. Acoustic frequency selective insulators and filters are possible applications.

Several computational methods have been adapted and developed to study wave propagation through PCs. Two such methods are the purely numerical finite-difference time domain (FDTD) method and the semianalytical layer multiple-scattering (LMS) method, which is developed from Korringa-Kohn-Rostoker theory [2]. These methods complement each other. Advantages with the LMS method are its computational speed, which makes it useful for forward modeling in connection with extensive optimization computations, and the physical insight it provides. It appears that the LMS method was first developed for the 3D case with spherical scatterers $[3,4]$, and recent review papers include Sigalas et al. [5] and Sainidou et al. [6].

The LMS method has also been applied to the 2D case with infinite cylindrical scatterers. It is mainly the inplane propagation case that has been considered [7-11], for which one space dimension can be eliminated in the wave equations. Out-of-plane propagation has been treated by Mei et al. [12] and, for band structure calculations, somewhat earlier by Wilm et al. [13] (using the plane-wave method) and by Guenneau et al. [14].

In the present paper, basic LMS equations for propagation of plane waves of any direction through a 2D PC slab are first provided in Section 2. The Poisson summation formula and the Graf addition theorem are utilized. As shown in Section 3, different types of scatterers at the same interface can be allowed, which represents an extension as compared to the treatment in [12]. Coupled equation systems are derived for the different scatterer types. For 3D PC slabs, a corresponding extension has recently proved useful for applications to design of anechoic coatings [15]. 


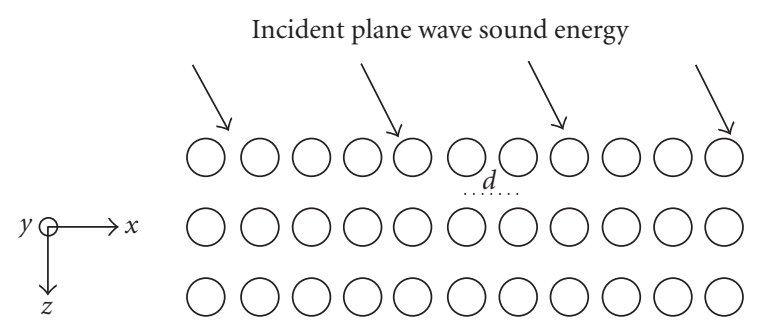

Figure 1: Horizontal $x y$ coordinates and a $z$ depth coordinate axis are introduced. There are three scatterer interfaces in this illustration. The medium is periodic with period $d$ in the $x$ direction, although only finitely many cylindrical scatterers are shown at each interface, and the cylinder axes are parallel to the $y$-axis.

Applications to design of 2D PC slabs with broad transmission gaps for incident waves of in-plane as well as out-of-plane directions are presented in Section 4. The design problem is equivalent to a nonlinear optimization problem, and differential evolution [16], a global optimization technique from inverse theory, is used. A 2D PC slab example from Mei et al. [12], with lead cylinders in an epoxy host, is revisited. Geometrical and material parameter values are varied to position and widen its band gap.

\section{Basic 2D Layer Multiple-Scattering Computational Method}

As in Figure 1, a right-hand Cartesian $x y z$ coordinate system is introduced in a fluid-solid medium surrounded by homogeneous half-spaces. The horizontal directions are $x$ and $y$. The medium is periodic with period $d$ in the $x$ direction and uniform in the $y$ direction.

Sound waves with time dependence $\exp (-i \omega t)$, to be suppressed in the formulas, are considered, where $\omega$ is the angular frequency. It follows that an incident plane wave with horizontal wavenumber vector $\mathbf{k}_{\|}=\left(k_{\|}, \kappa, 0\right)$ will give rise to a linear combination of reflected and transmitted plane waves with displacement vectors

$$
\mathbf{u}(\mathbf{r})=\exp \left(i \mathbf{K}_{\mathbf{g} j}^{s} \cdot \mathbf{r}\right) \cdot \mathbf{e}_{j} .
$$

Here, $\mathbf{r}=(x, y, z), j=1,2,3$ for a wave of type P,SV,SH, respectively, $s=+(-)$ for a wave in the positive (negative) $z$ direction, and

$$
\begin{aligned}
\mathbf{K}_{\mathbf{g} j}^{ \pm} & =\mathbf{k}_{\|}+\mathbf{g} \pm\left[\left(\frac{\omega}{c_{j}}\right)^{2}-\left|\mathbf{k}_{\|}+\mathbf{g}\right|^{2}\right]^{1 / 2} \cdot(0,0,1) \\
& =\frac{\omega}{c_{j}} \cdot(\sin \theta \cos \varphi, \sin \theta \sin \varphi, \cos \theta)
\end{aligned}
$$

where $\mathbf{g}$ belongs to the reciprocal lattice

$$
\mathbf{g}=\left(k_{x}, k_{y}, 0\right)=\left(\frac{2 \pi m}{d}, 0,0\right)
$$

with $m$ running over the integers. Furthermore, $c_{1}$ is the compressional-wave velocity $\alpha$ and $c_{2}=c_{3}$ are the shearwave velocity $\beta$. The angular variables $\theta, \varphi$ of $\mathbf{K}_{\mathbf{g} j}^{ \pm}$are defined by (2), with a possibly complex $\cos \theta$. The vectors $\mathbf{e}_{j}=$ $\mathbf{e}_{j}\left(\mathbf{K}_{\mathbf{g} j}^{ \pm}\right)$are defined by $\mathbf{e}_{1}=(\sin \theta \cos \varphi, \sin \theta \sin \varphi, \cos \theta)$, $\mathbf{e}_{2}=(\cos \theta \cos \varphi, \cos \theta \sin \varphi,-\sin \theta), \mathbf{e}_{3}=(-\sin \varphi, \cos \varphi, 0)$. It is convenient also to introduce the compressional- and shear-wave wavenumbers $k_{p}=\omega / \alpha$ and $k_{s}=\omega / \beta$.

As detailed in [17], for example, and references therein, reflection and transmission matrices $R_{B}, T_{B}$ and $R_{A}, T_{A}$ can now be introduced, for the discrete set of waves specified by (1)-(3). The mentioned reference concerns the doubly periodic case for a 3D PC, with periodicity in the $y$ direction as well, but the R/T matrix formalism is the same. There are three scatterer interfaces in the illustration of Figure 1. Individual $\mathrm{R} / \mathrm{T}$ matrices can be combined recursively [18, 19]. Layer thicknesses, as well as translations of individual scatterer interfaces in the $x$ direction, are conveniently accounted for by phase shifts of the complex amplitudes of the plane-wave components.

2.1. Interface with Periodically Distributed Scatterers of a Common Type. Explicit expressions for the R/T matrices are well known for an interface between two homogeneous half-spaces [19]. To handle an interface with periodically distributed scatterers, as one of the three in Figure 1, the following cylindrical vector solutions to the wave equations can be used [12]:

$$
\begin{gathered}
\mathbf{u}_{l \kappa}^{L}(\mathbf{r})=\frac{i}{k_{p}} \nabla\left[f_{l}(p r) \exp (i l \eta) \exp (i \kappa y)\right], \\
\mathbf{u}_{l \kappa}^{M}(\mathbf{r})=\frac{1}{k_{s}} \nabla \times \mathbf{u}_{l \kappa}^{N}(\mathbf{r}), \\
\mathbf{u}_{l \kappa}^{N}(\mathbf{r})=\frac{1}{k_{s}} \nabla \times\left[f_{l}(q r) \exp (i l \eta) \exp (i \kappa y) \mathbf{e}_{y}\right],
\end{gathered}
$$

where cylindrical coordinates $r, \eta, y$ are used according to $\mathbf{r}=(r \sin \eta, y, r \cos \eta)$, and $\mathbf{e}_{y}=(0,1,0)$. The index $l=$ $0, \pm 1, \pm \ldots$, and $\kappa$ is a real number. Residual wavenumbers $p, q$ are defined by

$$
p=\left(k_{p}^{2}-\kappa^{2}\right)^{1 / 2}, \quad q=\left(k_{s}^{2}-\kappa^{2}\right)^{1 / 2} .
$$

The notation $\mathbf{u}_{l \kappa}^{0 L}(\mathbf{r}), \mathbf{u}_{l \kappa}^{0 M}(\mathbf{r}), \mathbf{u}_{l \kappa}^{0 N}(\mathbf{r})$ and $\mathbf{u}_{l \kappa}^{+L}(\mathbf{r}), \mathbf{u}_{l \kappa}^{+M}(\mathbf{r})$, $\mathbf{u}_{l \kappa}^{+N}(\mathbf{r})$ is used for the two basic cases with $f_{l}$ as the Bessel function $J_{l}$ and $f_{l}$ as the Hankel function $H_{l}^{(1)}$, respectively.

For scatterers at

$$
\mathbf{R}=(x, y, z)=(m d, 0,0)
$$

where $d$ is the lattice period and $m$ runs over the integers, and for an incident plane wave as in (1), the total scattered field $\mathbf{u}_{\mathrm{sc}}$ can be written as (cf. [3])

$$
\begin{array}{r}
\mathbf{u}_{\mathrm{sc}}(\mathbf{r})=\sum_{P l}\left[b_{l}^{+P} \sum_{\mathbf{R}} \exp \left(i \mathbf{k}_{\|} \cdot \mathbf{R}\right) \cdot \mathbf{u}_{l \kappa}^{+P}(\mathbf{r}-\mathbf{R})\right], \\
P=L, M, N,
\end{array}
$$

where $\kappa$ is the $y$ component of $\mathbf{k}_{\|}$. 
The vector $\mathbf{b}^{+}=\left\{b_{l}{ }^{+P}\right\}$ is determined by solving the equation system

$$
(\mathbf{I}-T \cdot \Omega) \cdot \mathbf{b}^{+}=T \cdot \mathbf{a}^{0},
$$

where $\mathbf{I}$ is the appropriate identity matrix, $\mathbf{a}^{0}=\left\{a_{l}^{0 P}\right\}$ gives the coefficients for expansion of the incident plane wave in regular cylindrical waves $\mathbf{u}_{l k}^{O P}(\mathbf{r}), \Omega=\Omega\left(k_{\|} d, p d, q d\right)$ is the lattice translation matrix $\left\{\Omega_{l: l^{\prime}}^{P P^{\prime}}\right\}$, and $T=\left\{T_{l: l^{\prime}}^{P P^{\prime}}\right\}$ is the transition matrix for an individual scatterer. Specifically, $\mathbf{b}^{\prime}=\Omega \cdot \mathbf{b}^{+}$and $\mathbf{b}^{+}=T \cdot\left(\mathbf{a}^{0}+\mathbf{b}^{\prime}\right)$ where $\mathbf{b}^{\prime}=\left\{b_{l}^{\prime}\right\}^{2}$ gives the coefficients for expansion in regular cylindrical waves $\mathbf{u}_{l k}^{O P}(\mathbf{r})$ of the scattered field from all scatterers except the one at the origin.

The R/T matrices are obtained, finally, by transforming the expansion (9) to plane waves of the type (1). Specifically, (9) can be rewritten as

$$
\begin{aligned}
\mathbf{u}_{\mathrm{sc}}(\mathbf{r})=\sum_{\mathbf{g}} \sum_{j=1,2,3} \Delta & \Delta\left(d \mathbf{g}, j ; k_{\|} d, \kappa d, p d, q d, \mathbf{b}^{+}\right) \\
& \times \exp \left(i \mathbf{K}_{\mathrm{g} j}^{ \pm} \cdot \mathbf{r}\right) \cdot \mathbf{e}_{j} .
\end{aligned}
$$

The sign in $\mathbf{K}_{\mathbf{g} j}^{ \pm}$is given by the sign of $z$.

Explicit expressions for the $\Delta$ coefficients in (11) are readily obtained from (9) and (4)-(6) by invoking, for $k=p$ and $k=q$, the relation

$$
\begin{gathered}
\sum_{m} \exp \left(i m d k_{||}\right)\left[\frac{(z+i(x-m d))}{\left((x-m d)^{2}+z^{2}\right)^{1 / 2}}\right]^{l} \\
\times H_{l}^{(1)}\left(k\left((x-m d)^{2}+z^{2}\right)^{1 / 2}\right) \\
=2(-k)^{-l} \times \sum_{m}\left(\gamma_{m} d\right)^{-1}\left[-\left(k_{||}+\frac{2 \pi m}{d}\right)+i \gamma_{m} \operatorname{sgn}(z)\right]^{l} \\
\quad \times \exp \left[i\left(k_{||}+\frac{2 \pi m}{d}\right) x+i|z| \gamma_{m}\right],
\end{gathered}
$$

where $\gamma_{m}=\left[k^{2}-\left(k_{\|}+2 \pi m / d\right)^{2}\right]^{1 / 2}$ with $\operatorname{Im} \gamma_{m} \geq 0$. The relation (12) is valid for $z \neq 0$, and it can be verified using the Poisson summation formula.

2.2. Computation of the Expansion Coefficients $\mathbf{a}^{0}$, the Matrices $\Omega$, and the Matrices T. An incident plane compressional wave $\mathbf{u}_{\text {inc }}(\mathbf{r})=\exp \left(i k_{p} \mathbf{e}_{\text {inc }} \cdot \mathbf{r}\right) \mathbf{e}_{\text {inc }}$, where $\mathbf{e}_{\text {inc }}=$ $k_{p}^{-1}\left(p \sin \eta_{\mathrm{inc}}, \kappa, p \cos \eta_{\mathrm{inc}}\right)$, can be expanded as

$$
\mathbf{u}_{\text {inc }}(\mathbf{r})=-\sum_{l} \mathrm{i}^{l} \exp \left(-\mathrm{i} l \eta_{\text {inc }}\right) \mathbf{u}_{l \kappa}^{0 L}(\mathbf{r}) .
$$

Noting that $\mathbf{u}_{\text {inc }}(\mathbf{r})=\nabla\left[\exp \left(i k_{p} \mathbf{e}_{\text {inc }} \cdot \mathbf{r}\right)\right] / i k_{p}$, this follows readily from the well-known Bessel function relation $\exp (i \gamma \sin \eta)=\sum_{l} J_{l}(\gamma) \exp (i l \eta)$.

An incident plane shear wave of SV or SH type can be expanded by a superposition of two cases. The first case, $\mathbf{u}_{\text {inc }}(\mathbf{r})=\exp \left(i k_{s} \mathbf{e}_{\text {inc }} \cdot \mathbf{r}\right) \mathbf{e}_{\text {inc }}^{\perp}=-\left(k_{s} q\right)^{-1} \nabla \times\{\nabla \times$ $\left.\left[\exp \left(i k_{s} \mathbf{e}_{\text {inc }} \cdot \mathbf{r}\right) \mathbf{e}_{y}\right]\right\}$ with $\mathbf{e}_{\text {inc }}=k_{s}^{-1}\left(q \sin \eta_{\text {inc }}, \kappa, q \cos \eta_{\text {inc }}\right)$ and $\mathbf{e}_{\text {inc }}^{\perp}=k_{s}^{-1}\left(\kappa \sin \eta_{\text {inc }},-q, \kappa \cos \eta_{\text {inc }}\right)$, can be expanded as

$$
\mathbf{u}_{\text {inc }}(\mathbf{r})=-\frac{k_{s}}{q} \sum_{l} i^{l} \exp \left(-\mathrm{i} l \eta_{\text {inc }}\right) \mathbf{u}_{l k}^{0 M}(\mathbf{r}) .
$$

The second case, $\mathbf{u}_{\text {inc }}(\mathbf{r})=\exp \left(i k_{s} \mathbf{e}_{\text {inc }} \cdot \mathbf{r}\right) \mathbf{e}_{\text {inc }}^{\perp}=$ $i / q \nabla \times\left[\exp \left(i k_{s} \mathbf{e}_{\mathrm{inc}} \cdot \mathbf{r}\right) \mathbf{e}_{y}\right]$ with $\mathbf{e}_{\mathrm{inc}}$ as before and $\mathbf{e}_{\mathrm{inc}}^{\perp}=$ $\left(\cos \eta_{\text {inc }}, 0,-\sin \eta_{\text {inc }}\right)$, has the expansion

$$
\mathbf{u}_{\text {inc }}(\mathbf{r})=\frac{k_{s}}{q} \sum_{l} i^{l+1} \exp \left(-i l \eta_{\text {inc }}\right) \mathbf{u}_{l \kappa}^{0 N}(\mathbf{r})
$$

The lattice translation matrix $\Omega\left(k_{\|} d, p d, q d\right)$ can be determined by applying, for $k=p$ and $k=q$, the relation

$$
\begin{aligned}
& \sum_{m \neq 0} \exp \left(i m d k_{\|}\right)\left[\frac{(z+i(x-m d))}{\left((x-m d)^{2}+z^{2}\right)^{1 / 2}}\right]^{l} \\
& \times \mathrm{H}_{l}^{(1)}\left(k\left((x-m d)^{2}+z^{2}\right)^{1 / 2}\right) \\
& =\sum_{n}\left[\frac{(z+i x)}{\left(x^{2}+z^{2}\right)^{1 / 2}}\right]^{n} \Theta_{l-n}\left(k_{\|} d, k d\right) J_{n}(k r),
\end{aligned}
$$

where the last sum is taken over all integers $n$ and

$$
\begin{aligned}
\Theta_{l}\left(k_{\|} d, k d\right)= & i^{-l} \sum_{m>0} \exp \left(i m d k_{\|}\right) \mathrm{H}_{l}^{(1)}(m k d) \\
& +i^{l} \sum_{m>0} \exp \left(-i m d k_{\|}\right) \mathrm{H}_{l}^{(1)}(m k d) .
\end{aligned}
$$

This relation follows from the Graf addition theorem [20] for Bessel functions. The elements $\left\{\Omega_{l: l^{\prime}}^{P P^{\prime}}\right\}$ of the lattice translation matrix $\Omega\left(k_{\|} d, p d, q d\right)$ vanish unless $P=P^{\prime}$. The remaining elements depend on $l$ and $l^{\prime}$ through $\left|l-l^{\prime}\right|$ only. Explicitly,

$$
\begin{gathered}
\Omega_{l ; l^{\prime}}^{L L \prime^{\prime}}=\Theta_{l-l^{\prime}}\left(k_{\|} d, p d\right), \\
\Omega_{l ; l^{\prime}}^{M M^{\prime}}=\Omega_{l ; l^{\prime}}^{N N^{\prime}}=\Theta_{l-l^{\prime}}\left(k_{\|} d, q d\right) .
\end{gathered}
$$

Moroz [21] has published a representation of lattice sums in terms of exponentially convergent series, which has been used in the present work for numerical evaluation of the $\Theta$ quantities defined in (17).

For a homogeneous cylindrical scatterer, the interior field and the exterior field can be expanded in cylindrical waves $\mathbf{u}_{l \kappa}^{O P}(\mathbf{r})$ and $\mathbf{u}_{l \kappa}^{O P}(\mathbf{r}), \mathbf{u}_{l \kappa}^{+P}(\mathbf{r})$, respectively. An equation system for the $T$-matrix elements $T=\left\{T_{l: l^{\prime}}^{P P^{\prime}}\right\}$, which depend on $\kappa$, is then readily obtained from the standard boundary conditions concerning continuity of displacement and traction at the cylinder surface. The scatterer as well as the host medium can be either fluid or solid. Because of the circular symmetry with a cylindrical scatterer, scattering only appears to the same $l$ component $\left(l^{\prime}=l\right)$. Details concerning the case $\kappa=0$, for which the $P$-SV $\left(P, P^{\prime} \neq M\right)$ and $\mathrm{SH}$ $\left(P=P^{\prime}=M\right)$ solutions decouple, are given by Mei et al. [9]. 


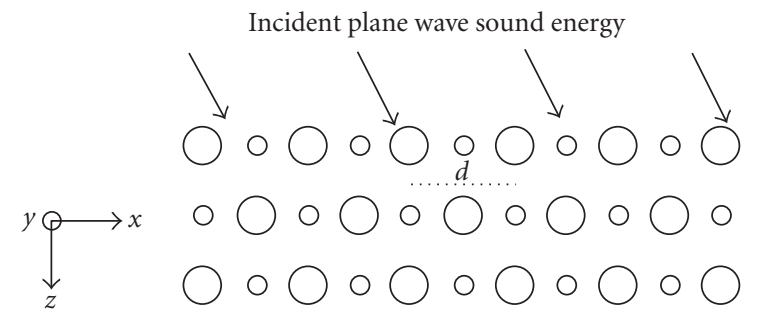

Figure 2: The configuration from Figure 1 is extended here, by allowing two types of cylindrical scatterers to appear in an alternating fashion at the same interface depth. The medium is periodic with period $d$ in the $x$ direction, and the cylinder axes are still parallel to the $y$-axis.

\section{Different Types of Scatterers at the Same Interface}

The LMS method is commonly applied for lattices with identical cylindrical scatterers within the same layer. As shown below, however, different types of scatterers within the same layer can also be accommodated. A similar extension for the restriction to in-plane wave propagation is made in Ivansson [22].

An illustration is given in Figure 2. Centered at the same $z$ level, at each of the three scatterer interfaces, there are two types of cylindrical scatterers. Each scatterer interface is treated separately. Choosing coordinates appropriately, scatterers of the first type, with transition matrix $T$ and scattered-field expansion coefficients denoted $\mathbf{b}^{+}$, appear at $\mathbf{R}=m \cdot(d, 0,0)$, for integers $m$. Scatterers of the second type, with transition matrix $U$ and scattered-field expansion coefficients denoted $c^{+}$, appear at points $S$ in between, that is, $\mathbf{S}=(m+1 / 2) \cdot(d, 0,0)$. The reciprocal lattice vectors become $\mathbf{g}=(2 \pi m / d, 0,0)$, where $m$ runs over the integers.

The generalization of the expression (9) for the scattered field becomes

$$
\begin{aligned}
\mathbf{u}_{\mathrm{sc}}(\mathbf{r})= & \sum_{P l}\left[b_{l}^{+P} \sum_{\mathbf{R}} \exp \left(i \mathbf{k}_{\|} \cdot \mathbf{R}\right) \cdot \mathbf{u}_{l \kappa}^{+P}(\mathbf{r}-\mathbf{R})\right] \\
& +\sum_{P l}\left[c_{l}^{+P} \sum_{\mathbf{S}} \exp \left(i \mathbf{k}_{\|} \cdot \mathbf{S}\right) \cdot \mathbf{u}_{l \kappa}^{+P}(\mathbf{r}-\mathbf{S})\right] .
\end{aligned}
$$

It follows that

$$
\mathbf{b}^{+}=T \cdot\left(\mathbf{a}^{0}+\mathbf{b}^{\prime}+\mathbf{b}^{\prime \prime}\right), \quad \mathbf{c}^{+}=U \cdot\left(\mathbf{a}^{0}+\mathbf{c}^{\prime}+\mathbf{c}^{\prime \prime}\right)
$$

where, for a scatterer of the first type at $\mathbf{R}, \exp \left(i \mathbf{k}_{\|} \cdot \mathbf{R}\right) \mathbf{b}^{\prime}$ and $\exp \left(i \mathbf{k}_{\|} \cdot \mathbf{R}\right) \mathbf{b}^{\prime \prime}$ give the coefficients for expansion in regular cylindrical waves $\mathbf{u}_{l \kappa}^{O P}(\mathbf{r}-\mathbf{R})$ of the scattered field from all other scatterers of the first and the second types, respectively. The vectors $\mathbf{c}^{\prime}$ and $\mathbf{c}^{\prime \prime}$ are defined analogously. For a scatterer of the second type at $\mathbf{S}, \exp \left(i \mathbf{k}_{\|} \cdot \mathbf{S}\right) \mathbf{c}^{\prime}$ and $\exp \left(\mathbf{i} \mathbf{k}_{\|} \cdot \mathbf{S}\right) \mathbf{c}^{\prime \prime}$ thus give the coefficients for expansion in regular cylindrical waves $\mathbf{u}_{l \kappa}{ }^{O P}(\mathbf{r}-\mathbf{S})$ of the scattered field from all other scatterers of the second and the first types, respectively.
With $\Omega^{0}=\Omega\left(k_{\|} d, p d, q d\right)$, it follows that $\mathbf{b}^{\prime}=\Omega^{0} \cdot \mathbf{b}^{+}$ and $\mathbf{c}^{\prime}=\Omega^{0} \cdot \mathbf{c}^{+}$. For a certain matrix $\Omega^{\text {dif }}$, to be determined, $\mathbf{b}^{\prime \prime}=\Omega^{\text {dif }} \cdot \mathbf{c}^{+}$and $\mathbf{c}^{\prime \prime}=\Omega^{\text {dif }} \cdot \mathbf{b}^{+}$. The equation system for determination of $\mathbf{b}^{+}$and $\mathbf{c}^{+}$becomes

$$
\begin{gathered}
\left(\mathbf{I}-T \cdot \Omega^{0}\right) \cdot \mathbf{b}^{+}-T \cdot \Omega^{\mathrm{dif}} \cdot \mathbf{c}^{+}=T \cdot \mathbf{a}^{0}, \\
-U \cdot \Omega^{\mathrm{dif}} \cdot \mathbf{b}^{+}+\left(\mathbf{I}-U \cdot \Omega^{0}\right) \cdot \mathbf{c}^{+}=U \cdot \mathbf{a}^{0} .
\end{gathered}
$$

In order to form the $\mathrm{R} / \mathrm{T}$ matrices, incident plane waves with different horizontal wavenumber vectors $\mathbf{k}_{\|}+\mathbf{g}_{\text {inc }}=$ $\left(k_{\|}+g_{\text {inc }}, \kappa, 0\right)$ have to be considered, where $g_{\text {inc }}$ belongs to the set $\{2 \pi \mathrm{m} / d\}$ providing the reciprocal lattice. Noting that the union of the scatterer positions is a small square lattice with period $d / 2$, the following expression for $\Omega^{\text {dif }}$ as a difference of $\Omega$ matrices is directly obtained

$$
\Omega^{\mathrm{dif}}=\Omega\left(\frac{\left(k_{\|}+g_{\text {inc }}\right) d}{2}, \frac{p d}{2}, \frac{q d}{2}\right)-\Omega^{0} .
$$

Only those $\mathbf{g}_{\text {inc }}$ in $\{(2 \pi m / d, 0,0)\}$ for which $m$ is even are reciprocal vectors for the small lattice with period $d / 2$. Since a lattice translation matrix $\Omega$ is periodic in its first argument with period $2 \pi$, there will be two groups of $\mathbf{g}_{\text {inc }}$ with different $\Omega^{\mathrm{dif}}$ matrices according to (22). Specifically,

$$
\Omega^{\text {dif,even }}=\Omega\left(\frac{k_{\|} d}{2}, \frac{p d}{2}, \frac{q d}{2}\right)-\Omega^{0}
$$

pertains to $\mathbf{g}_{\text {inc }}=(2 \pi m / d, 0,0)$ with even $m$, and

$$
\Omega^{\mathrm{dif}, \mathrm{odd}}=\Omega\left(\frac{k_{\|} d}{2}+\pi, \frac{p d}{2}, \frac{q d}{2}\right)-\Omega^{0}
$$

pertains to $\mathbf{g}_{\text {inc }}=(2 \pi m / d, 0,0)$ with odd $m$.

The transformation of the expansion (19) to plane waves of the type (1) can be done separately for each of the $\mathbf{R}$ and $\mathbf{S}$ sums. In the latter case, the translation from the origin causes a sign change for some combinations of incident $\left(\mathbf{g}_{\text {inc }}\right)$ and scattered $\left(\mathbf{g}_{\mathrm{sc}}\right)$ reciprocal lattice vectors. Specifically, with $\mathbf{g}_{\text {inc }}=\left(2 \pi m_{\text {inc }} / d, 0,0\right)$ and $\mathbf{g}_{\mathrm{sc}}=\left(2 \pi m_{\mathrm{sc}} / d, 0,0\right)$, the double sum corresponding to the one in (11) appears as

$$
\begin{gathered}
\mathbf{u}_{\mathrm{sc}}(\mathbf{r})=\sum_{\mathbf{g}_{\mathrm{sc}}} \sum_{j=1,2,3}(-1)^{m_{\mathrm{inc}}-m_{\mathrm{sc}}} \Delta\left(d \mathbf{g}_{\mathrm{sc}}, j ; k_{\|} d, \kappa d, p d, q d, \mathbf{c}^{+}\right) \\
\quad \times \exp \left(i \mathbf{K}_{\mathbf{g}_{\mathrm{sc}}}^{ \pm} \cdot \mathbf{r}\right) \cdot \mathbf{e}_{j} .
\end{gathered}
$$

\section{Designing 2D PC Slabs with a Transmission Gap}

It is well known that band gaps can appear when scatterers with a large density are arranged periodically in a host with a small density. A particular 2D PC slab example with lead cylinders in epoxy was considered in Mei et al. [12]. The epoxy parameters were 2540 and $1159.817 \mathrm{~m} / \mathrm{s}$ for the compressional- and shear-wave velocities, respectively, and $1.18 \mathrm{~kg} / \mathrm{dm}^{3}$ for the density. Corresponding lead material parameters were 2160 and $860.568 \mathrm{~m} / \mathrm{s}$, and $11.4 \mathrm{~kg} / \mathrm{dm}^{3}$. 


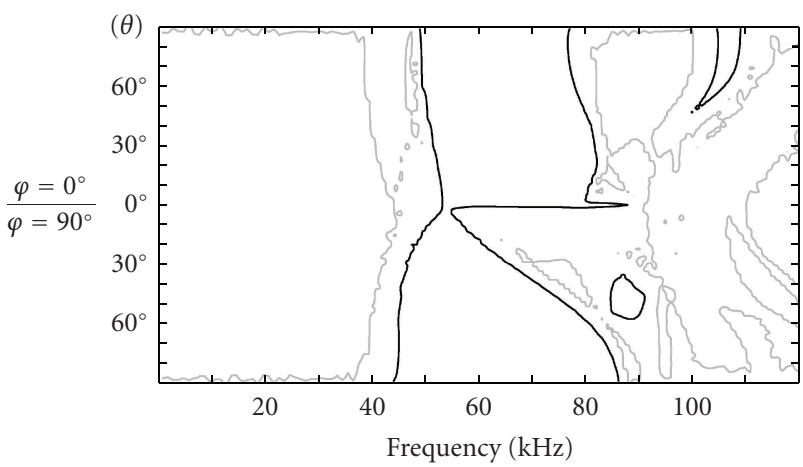

Figure 3: Contour plot of transmittance for a PC slab with lead cylinders in an epoxy host. Parameters of the slab are given in the text. The direction vector of the incident compressional wave is ( $\sin$ $\theta \cos \varphi, \sin \theta \sin \varphi, \cos \theta)$. The black and gray contours are at $-150 \mathrm{~dB}$ and $-10 \mathrm{~dB}$, respectively.

The slab was formed by sixteen layers of lead cylinders, each with a radius of $3.584 \mathrm{~mm}$, with a spacing between cylinder centers of $11.598 \mathrm{~mm}$ in the $x$ as well as $z$ directions, cf. Figure 1 where there are three layers. A band gap centered at about $60 \mathrm{kHz}$ was found. (Dimensionless frequencies and distances were actually used in the paper, but a specialization is made here.)

Figure 3 is a contour plot of the transmittance, that is, time- (and space-) averaged transmitted energy flux relative to the incident one, up to $120 \mathrm{kHz}$. The vertical axis is for the angle $\theta$, where the direction vector of the incident compressional plane wave is $(\sin \theta \cos \varphi, \sin \theta \sin \varphi, \cos \theta)$. In-plane and out-of-plane incidence angles are considered together in Figure 3, where the upper half with $\varphi=0^{\circ}$ concerns incidence in the $x z$ plane (in-plane propagation) and the lower half with $\varphi=90^{\circ}$ concerns incidence in the $y z$ plane. Only the first Brillouin zone is involved, since $k_{x}=\omega / \alpha \sin \theta \cos \varphi<2 \pi / d$ when $\alpha$ equals the epoxy compressional-wave velocity $2540 \mathrm{~m} / \mathrm{s}, d=11.598 \mathrm{~mm}$, and the frequency is less than $\alpha / d=219 \mathrm{kHz}$. Of course, the band gap from Mei et al. [12], at about $60 \mathrm{kHz}$, shows up clearly in this kind of plot. One might wonder how the geometrical and/or material parameters of the slab should be modified to achieve a prescribed desired change of the appearance of the gap.

Global optimization methods can be used to design PC slabs with desirable properties. Simulated annealing, genetic algorithms and differential evolution (DE) are three kinds of such methods, that have become popular during the last fifteen years. DE, to be applied here, is related to genetic algorithms, but the parameters are not encoded in bit strings, and genetic operators such as crossover and mutation are replaced by algebraic operators [16].

As a very simple example, to try to position and widen the gap in Figure 3, an objective function for DE minimization is specified as the maximum transmittance in the frequency band $45-65 \mathrm{kHz}$ when the incidence angle $\theta$ is varied between $0^{\circ}$ and $20^{\circ}$ for the two azimuthal angles $\varphi=0^{\circ}$ (inplane propagation in the $x z$ plane) and $\varphi=90^{\circ}$ (propagation

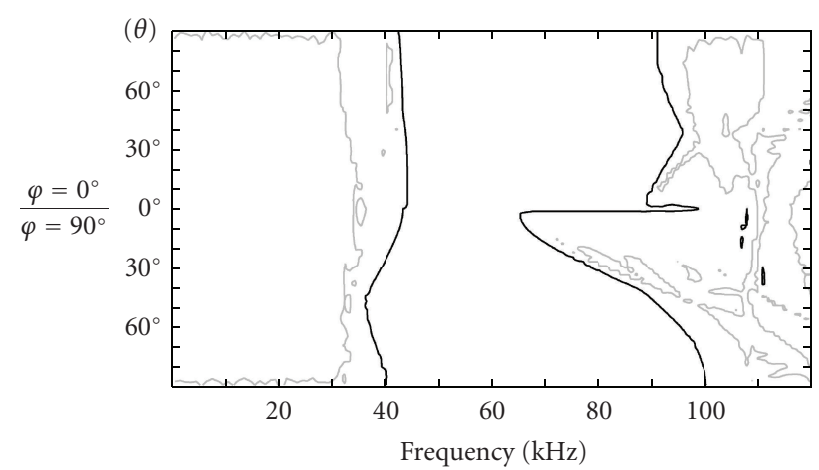

Figure 4: Contour plot of transmittance as in Figure 3 but for the optimized PC slab specified in Table 1.

TABle 1: Specification of a PC slab that has been optimized by $\mathrm{DE}$ to produce small transmittance in the band $45-65 \mathrm{kHz}$. Corresponding transmittance results are shown in Figures 4 and 5.

\begin{tabular}{lc}
\hline Optimum & $-163.0 \mathrm{~dB}$ \\
\hline Scatterer compressional-wave velocity $\alpha$ & $2995.6 \mathrm{~m} / \mathrm{s}$ \\
Scatterer shear-wave velocity $\beta$ & $1200.0 \mathrm{~m} / \mathrm{s}$ \\
Scatterer density $\rho$ & $14.000 \mathrm{~kg} / \mathrm{dm}^{3}$ \\
Layer thickness $h$ & $13.292 \mathrm{~mm}$ \\
Largest scatterer radius $r_{\max }$ & $4.2702 \mathrm{~mm}$ \\
Smallest scatterer radius $r_{\min }$ & $4.2300 \mathrm{~mm}$ \\
Lattice period $d$ & $22.670 \mathrm{~mm}$ \\
\hline
\end{tabular}

in the $y z$ plane). A configuration with cylinders of two alternating sizes is allowed, as depicted in Figure 2. Still, the slab is formed by sixteen layers of cylinders. The following seven parameters are varied within the indicated search space: scatterer compressional-wave velocity $\alpha[1500 \mathrm{~m} / \mathrm{s} \leq$ $\alpha \leq 3000 \mathrm{~m} / \mathrm{s}]$, scatterer shear-wave velocity $\beta[650 \mathrm{~m} / \mathrm{s}$ $\leq \beta \leq 1200 \mathrm{~m} / \mathrm{s}]$, scatterer density $\rho\left[7 \mathrm{~kg} / \mathrm{dm}^{3} \leq \rho \leq\right.$ $\left.14 \mathrm{~kg} / \mathrm{dm}^{3}\right]$, layer thickness $h[8 \leq h \leq 16 \mathrm{~mm}]$, the largest scatterer radius $r_{\max }\left[0.225 h \leq r_{\max } \leq 0.4 h\right]$, the smallest scatterer radius $r_{\min }\left[0.45 r_{\max } \leq r_{\min } \leq r_{\max }\right]$, the lattice period $d\left[8\left(r_{\max }+r_{\min }\right) / 3 \leq d \leq 4\left(r_{\max }+r_{\min }\right)\right]$. The layer thickness $h$ is the $z$ distance between subsequent scatterer interfaces (cf. Figure 2).

Table 1 shows the optimum obtained with DE, along with corresponding parameter values. A reduction of the transmitted field with more than $160 \mathrm{~dB}$ is achieved throughout the band $45-65 \mathrm{kHz}$ and throughout the solid angle intervals $0^{\circ}<\theta<20^{\circ}$ for $\varphi=0^{\circ}, \varphi=90^{\circ}$ for the direction of incidence. High-velocity high-density cylinders seem preferable, since the lead velocities and density are all increased to values close to the upper ends of the corresponding search intervals. In this case, as large as possible values of $\alpha, \beta$, and $\rho$ could in fact have been fixed from the start. Moreover, the cylinders are almost as densely packed horizontally as allowed by the search interval for $d$. Hence, the optimization could in fact have been simplified considerably. With only a few free parameters, a complete search, for example, can be a feasible alternative. 


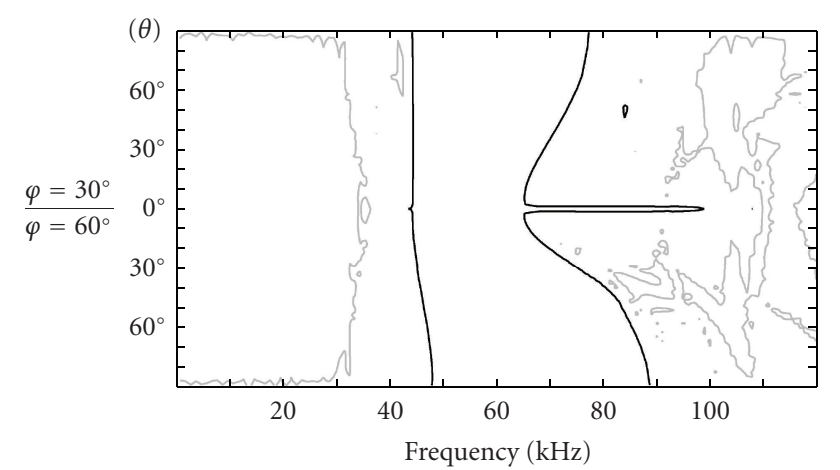

Figure 5: Contour plot of transmittance for the optimized PC slab specified in Table 1 . The difference to Figure 4 is that two other $\varphi$ angles are considered for the direction of the incident compressional wave.

Compared to Figure 3, the contour plot in Figure 4 of the transmittance for the optimized PC slab shows a band gap around $60 \mathrm{kHz}$ that has become significantly wider and also deeper. The allowed increases of $\alpha, \beta, \rho$, and $\left(r_{\max }+r_{\min }\right) / d$ are certainly essential for producing this effect. Variation of $h, r_{\max }$, and $r_{\min }$ is needed to position the band gap at the desired frequency interval. Although the optimization was performed with the restriction $0^{\circ}<\theta<20^{\circ}$, small transmittance is apparently achieved for all angles $\theta\left(0^{\circ}<\right.$ $\left.\theta<90^{\circ}\right)$.

It turns out that the transmittance for the optimized PC slab remains small within the $45-65 \mathrm{kHz}$ band for all outof-plane incidence angles (an "absolute" band gap). Figure 5 shows the transmittance in the same way as Figure 4, but for $\varphi=0^{\circ}$ changed to $\varphi=30^{\circ}$ and $\varphi=90^{\circ}$ changed to $\varphi=$ $60^{\circ}$. The upper and lower halves exhibit increased symmetry, since the $\varphi$ angles involved are closer to one another.

Apparently, the optimized PC slab has large transmittance below about $30 \mathrm{kHz}$ and there are regions with large transmittance above $80 \mathrm{kHz}$ as well. The band gap with small transmittance extends to higher frequencies, than those in the band $45-65 \mathrm{kHz}$, for plane-wave incidence directions with either large $\theta$ or small $\varphi$.

The efficacy of the DE technique can be illustrated by showing the decrease of the maximum transmittance within the specified frequency/angle region as the number of tested parameter settings for the PC slab evolves. Figure 6 shows this decrease for the example from Figure 4. A comparison to the much less efficient MonteCarlo method, with random selection of parameters from the search space, is included. The low efficiency of the MonteCarlo approach shows that the desired PC slabs with small transmittance only appear in a small portion of the search space. For example, only about $0.15 \%$ of the random slab selections had a maximum transmittance below $-20 \mathrm{~dB}$ within the specified frequency/angle region. About $0.01 \%$ had a maximum transmittance below $-100 \mathrm{~dB}$.

The difference between $r_{\max }$ and $r_{\min }$ in Table 1 is rather small. Optimization was also tried with all cylinders of exactly the same radius, as in Figure 1. The obtained optimum, with cylinders of radius $4.2503 \mathrm{~mm}$ and slightly

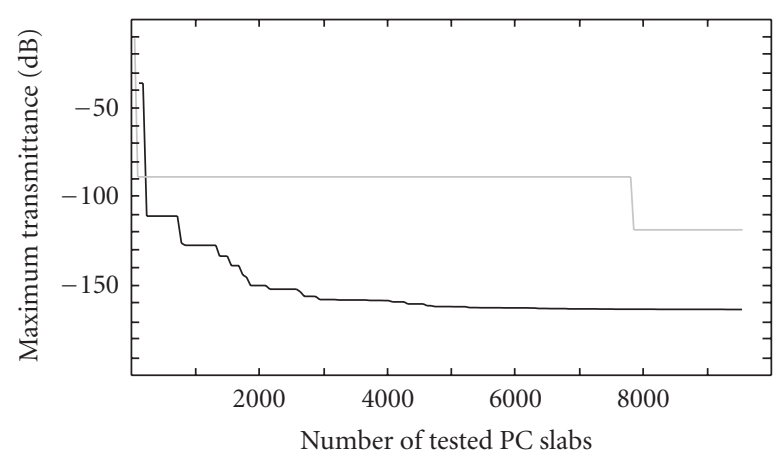

FIGURE 6: Evolution of the maximum transmittance with the number of tested parameter combinations for the DE optimization leading to the PC slab specified in Table 1. A corresponding curve for a brute force Monte Carlo method is also included (gray).

modified values for the other parameters, was almost as good as the one presented in Table 1. The advantages with cylinders of different sizes are expected to be more significant in more complicated filtering cases, involving, for example, more than one frequency band.

\section{Conclusions}

The layer multiple-scattering (LMS) method is a fast semianalytical technique for computing scattering from layers including periodic scatterer lattices. For the $2 \mathrm{D}$ case with cylindrical scatterers and any solid angle direction of an incident plane wave, an extension has been made to scatterer lattices with cylindrical scatterers of two different sizes in the same horizontal plane.

Global optimization methods from inverse theory are useful for designing PC slabs with desirable properties. A differential evolution algorithm has been applied here to position and widen an "absolute" band gap for a certain 2D PC slab. Although only limited angle intervals for the direction of incidence were included for the optimization, small transmittance was achieved for all solid angles specifying the direction of the incident wave.

The possibility to include cylindrical scatterers of two different sizes in the same horizontal plane provides an additional degree of freedom that can be useful for PC design purposes. In the presented example, with its specification of objective function and search intervals for the parameters, however, the difference between the optimal cylinder radii was rather small and the improvement only marginal. The additional flexibility is expected to be more important in more complicated filtering applications. For example, specified regions in frequency-angle space with large transmittance could be desired in addition to specified regions with small transmittance.

\section{Acknowledgment}

Alexander Moroz kindly provided his Fortran routine for calculation of lattice sums. 


\section{References}

[1] T. Miyashita, "Sonic crystals and sonic wave-guides," Measurement Science and Technology, vol. 16, no. 5, pp. R47-R63, 2005.

[2] N. Papanikolaou, R. Zeller, and P. H. Dederichs, "Conceptual improvements of the KKR method," Journal of Physics: Condensed Matter, vol. 14, no. 11, pp. 2799-2823, 2002.

[3] I. E. Psarobas, N. Stefanou, and A. Modinos, "Scattering of elastic waves by periodic arrays of spherical bodies," Physical Review B, vol. 62, no. 1, pp. 278-291, 2000.

[4] Z. Liu, C. T. Chan, P. Sheng, A. L. Goertzen, and J. H. Page, "Elastic wave scattering by periodic structures of spherical objects: theory and experiment," Physical Review B, vol. 62, no. 4, pp. 2446-2457, 2000.

[5] M. Sigalas, M. S. Kushwaha, E. N. Economou, M. Kafesaki, I. E. Psarobas, and W. Steurer, "Classical vibrational modes in phononic lattices: theory and experiment," Zeitschrift für Kristallographie, vol. 220, no. 9-10, pp. 765-809, 2005.

[6] R. Sainidou, N. Stefanou, I. E. Psarobas, and A. Modinos, "The layer multiple-scattering method applied to phononic crystals," Zeitschrift für Kristallographie, vol. 220, no. 9-10, pp. 848-858, 2005.

[7] S. B. Platts, N. V. Movchan, R. C. McPhedran, and A. B. Movchan, "Two-dimensional phononic crystals and scattering of elastic waves by an array of voids," Proceedings of the Royal Society of London A, vol. 458, no. 2026, pp. 2327-2347, 2002.

[8] S. B. Platts, N. V. Movchan, R. C. McPhedran, and A. B. Movchan, "Transmission and polarization of elastic waves in irregular structures," Transactions of the ASME, vol. 125, no. 1, pp. 2-6, 2003.

[9] J. Mei, Z. Liu, J. Shi, and D. Tian, “Theory for elastic wave scattering by a two-dimensional periodic array of cylinders: an ideal approach for band-structure calculations," Physical Review B, vol. 67, Article ID 245107, 7 pages, 2003.

[10] C. Qiu, Z. Liu, J. Mei, and M. Ke, "The layer multiplescattering method for calculating transmission coefficients of 2D phononic crystals," Solid State Communications, vol. 134, no. 11, pp. 765-770, 2005.

[11] S. Robert, J.-M. Conoir, and H. Franklin, "Propagation of elastic waves through two-dimensional lattices of cylindrical empty or water-filled inclusions in an aluminum matrix," Ultrasonics, vol. 45, no. 1-4, pp. 178-187, 2006.

[12] J. Mei, Z. Liu, and C. Qiu, "Multiple-scattering theory for out-of-plane propagation of elastic waves in two-dimensional phononic crystals," Journal of Physics: Condensed Matter, vol. 17, no. 25, pp. 3735-3757, 2005.

[13] M. Wilm, A. Khelif, S. Ballandras, V. Laude, and B. DjafariRouhani, "Out-of-plane propagation of elastic waves in twodimensional phononic band-gap materials," Physical Review E, vol. 67, no. 6, Article ID 065602, 4 pages, 2003.

[14] S. Guenneau, C. G. Poulton, and A. B. Movchan, "Oblique propagation of electromagnetic and elastic waves for an array of cylindrical fibres," Proceedings of the Royal Society of London A, vol. 459, no. 2037, pp. 2215-2263, 2003.

[15] S. M. Ivansson, "Numerical design of Alberich anechoic coatings with superellipsoidal cavities of mixed sizes," Journal of the Acoustical Society of America, vol. 124, no. 4, pp. 19741984, 2008.

[16] K. Price, R. Storn, and J. Lampinen, Differential EvolutionA Practical Approach to Global Optimization, Springer, New York, NY, USA, 2005.

[17] S. M. Ivansson, "Sound absorption by viscoelastic coatings with periodically distributed cavities," Journal of the Acoustical Society of America, vol. 119, no. 6, pp. 3558-3567, 2006.
[18] J. B. Pendry, Low Energy Electron Diffraction, Academic Press, New York, NY, USA, 1974.

[19] B. L. N. Kennett, Seismic Wave Propagation in Stratified Media, Cambridge University Press, Cambridge, UK, 1983.

[20] M. Abramowitz and I. A. Stegun, Handbook of Mathematical Functions, National Bureau of Standards, 1965.

[21] A. Moroz, "Exponentially convergent lattice sums," Optics Letters, vol. 26, no. 15, pp. 1119-1121, 2001.

[22] S. M. Ivansson, "Extended layer multiple-scattering and global optimization techniques for 2D phononic crystal insulator design," in Photonics Research Developments, V. P. Nilsson, Ed., chapter 7, pp. 225-245, Nova, 2008. 

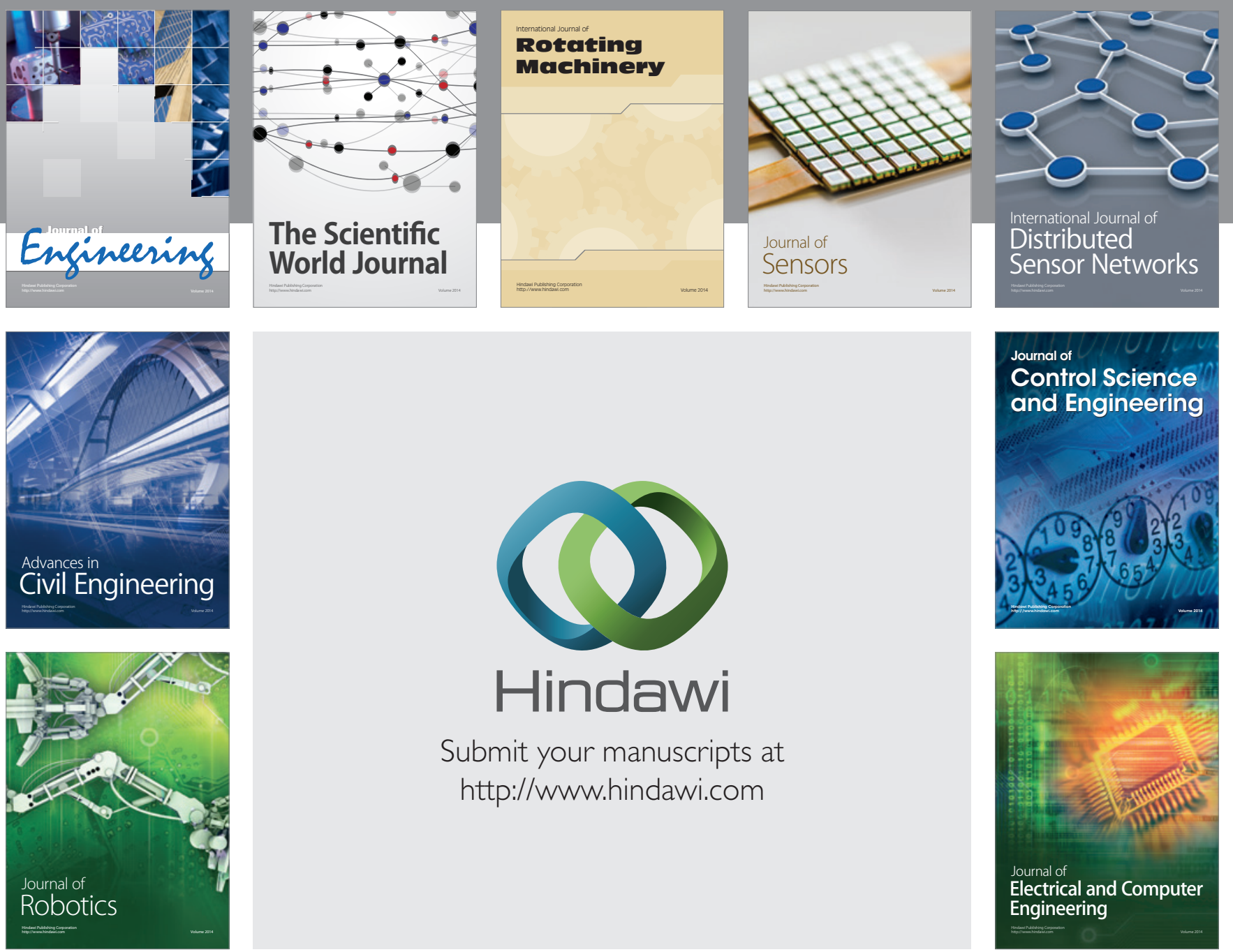

Submit your manuscripts at

http://www.hindawi.com
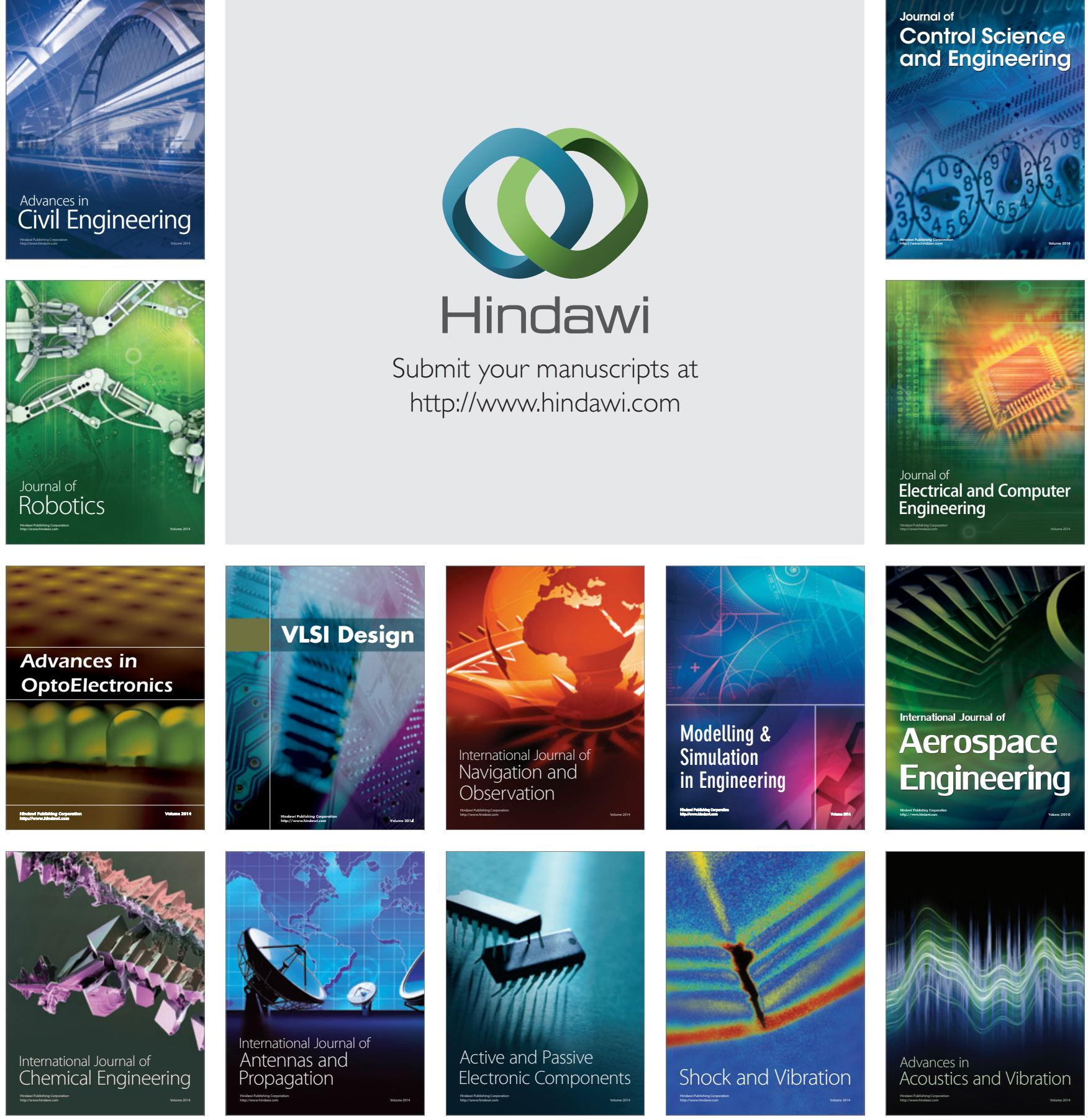